\title{
Assembly of Linked Nanocrystal Colloids by Reversible Covalent Bonds
}

\section{Supporting Information}

Manuel N. Dominguez ${ }^{1}$, Michael P. Howard ${ }^{2, \dagger}$, Josef M. Maier ${ }^{1, \dagger}$, Stephanie A. Valenzuela ${ }^{1, \neq}$, Zachary M. Shermann ${ }^{2, \ddagger}$, James F. Reuther ${ }^{1, \&}$, Lauren C. Reimnitz ${ }^{2}$, ${ }^{2}$ Jiho Kang ${ }^{2}$, ${ }^{2}$ Shin Hum Cho ${ }^{2, \#, ~ S t e p h e n ~ L . ~ G i b b s ~}{ }^{2}$, Arjun K. Menta ${ }^{1}$, Deborah L. Zhuang ${ }^{1}$, Aevi van der Stok ${ }^{1}$, Sarah J. Kline ${ }^{2}$, Eric V. Anslyn ${ }^{1 *}$, Thomas M. Truskett ${ }^{2,3^{*}}$, Delia J. Milliron ${ }^{1,2 *}$

${ }^{1}$ Department of Chemistry, University of Texas at Austin, Austin, Texas, 78712 1589

2 McKetta Department of Chemical Engineering, University of Texas at Austin, Austin, Texas, 78712 - 1589

${ }^{3}$ Department of Physics, University of Texas at Austin, Austin, Texas, 78712 1589

†These authors contributed equally.

¥These authors contributed equally.

\&Current address: Department of Chemistry, University of Massachusetts Lowell, Lowell, MA 01854

\#Current address: Samsung Electronics, Samsung Semiconductor R\&D, Hwaseong, Gyeonggi-do 18448, Republic of Korea

${ }^{*}$ Corresponding Authors

Email: anslyn@austin.utexas.edu, truskett@che.utexas.edu, milliron@che.utexas.edu 
Table of Contents

EXPERIMENTAL MATERIALS AND METHODS

S1. LCMS of $A L$ and $B L$

S2. SAXS NC Sizing and zeta potential measurement

S3. FTIR of NC and ligand fingerprint region

S4. ${ }^{19} \mathrm{~F}-\mathrm{NMR}$ of the AL capped ITO NCs

S5. TGA of oleate and TORC capped NCs

S6. DLS of initial assembly observations

S7. Photos of gel/cluster/dispersed samples

S8. SAXS structure factor for low $\Gamma$ samples

59. Photo and SAXS of control NC gelation experiment

S10. Porod region fitting for $\Gamma=100$ and $\Gamma=200$

S11. FTIR of AL-NC before/after assembly

S12. STEM of assembled NCs

\section{THEORETICAL MODEL AND METHODS}

S13. Schematic of NC with DCB ligands and linker molecules

S14. Low- $\Gamma$ branch of the spinodal boundary for $\mathrm{NC}$-linker mixture with varying percentages of functionalized ligands

S15. Analysis of simulated NC-linker structures with limited NC functionalization

S16. Fraction of linkers with different numbers of bonds for NCs with limited functionalization

\section{REFERENCES}




\section{EXPERIMENTAL METHODS}

Materials. Indium(III) acetate $\left(\operatorname{In}(\mathrm{ac})_{3}, 99.99 \%\right)$, Tin (IV) acetate $\left(\mathrm{Sn}(\mathrm{ac})_{4},>99.9 \%\right)$, oleic acid (OA, 90\%, technical grade), and oleyl alcohol (OleOH, 98\%) were purchased from Sigma-Aldrich. Dimethylformamide (99\%), Hexane (99.8\%), ethanol $(95.0 \%)$, and toluene $(99.5 \%)$ were purchased from Fisher Chemical. All deuterated solvents were purchased from Sigma Aldrich. All chemicals were used as received without any further purification.

Tin-Doped Indium Oxide (Sn:In $\mathrm{O}_{3}$ (ITO)) Nanocrystal Synthesis. All synthesis procedures were carried out on a Schlenk line. NC synthesis was carried out by modification of methods published by the Hutchison group. ${ }^{1}$ NCs were synthesized by adding $5.0 \mathrm{M}$ of metal precursor $(4.75 \mathrm{mmol}$ In(III)acetate, $0.25 \mathrm{mmol}$ $\mathrm{Sn}(\mathrm{IV})$ acetate) in oleic acid $(10 \mathrm{~mL}, \mathrm{OA})$ to a round bottom flask (precursor solution). The precursor solution is put under vacuum, stirred, and heated $\left(100{ }^{\circ} \mathrm{C}\right)$ for 1 hour with a nitrogen purge at the end of this vacuum step. The precursor solution is then kept under flowing nitrogen and heated $\left(150{ }^{\circ} \mathrm{C}\right)$ for 2 hours to generate In- and Snoleate. Simultaneously, a second round bottom flask is loaded with $13 \mathrm{~mL}$ oleyl alcohol $(\mathrm{OleOH})$. The OleOH is put under vacuum and heated $\left(100{ }^{\circ} \mathrm{C}\right)$ for 2 hours and purged in between this time. The $\mathrm{OleOH}$ is then heated $\left(290^{\circ} \mathrm{C}\right)$ under nitrogen. Once the precursor solution reactions are completed, the precursor solution is loaded into a glass syringe for a slow injection into the $\mathrm{OleOH}$ round bottom creating our reaction mixture. The injection rate is set to $0.2 \mathrm{~mL} / \mathrm{min}$ and the injection volume determines the NC size at the end of synthesis; in this case $3 \mathrm{~mL}$ was injected. The reaction mixture is then kept at the reaction temperature $\left(290{ }^{\circ} \mathrm{C}\right)$ for 10 minutes before being cooled to room temperature. The NCs were washed by 5 cycles of flocculating the NCs with ethanol, centrifuging at 7500 RPM, and redispersing in hexane. Size and polydispersity were measure by small-angle X-ray scattering (SAXS) and verified by scanning transmission electron microscopy (STEM). Ligand shells were characterized by ${ }^{1} \mathrm{H}$ nuclear magnetic resonance (NMR) and Fourier transform infrared (FTIR) spectroscopy analysis.

Aldehyde-Functionalized and Benzyl-Functionalized Ligand ( $A L$ and $B L$ ) Synthesis. The ligands were made using microwave assisted solid-phase peptide synthesis (SPPS) followed by solid-phase copper-catalyzed azide alkyne cycloaddtion (SP-CuAAC) to add the dynamic covalent bonding functionality. The microwave solid phase peptide synthesis was conducted using a CEM Liberty Blue Automated Microwave Peptide Synthesizer at $0.25 \mathrm{mmol}$ scales. Microwave-SPPS was conducted using the standard Fmoc/tBu protection strategy. All ligands were synthesized using d Wang resin (Fmoc-Asp(OtBu)-Wang, $0.51 \mathrm{mmol} / \mathrm{g}$ ). The microwave coupling performed using $\mathrm{DICl}$ Oxyma $\left(90^{\circ} \mathrm{C}\right.$ for $\left.110 \mathrm{~s}\right)$. Fmocdeprotections of amines were performed using 20 vol\% piperidine in DMF in two stages $\left(75^{\circ} \mathrm{C}\right.$ and $155 \mathrm{~W}$ for $15 \mathrm{~s}$ followed by $90^{\circ} \mathrm{C}$ for $\left.50 \mathrm{~s}\right)$. The resulting resin was washed with DMF $(5 \times 10 \mathrm{~mL})$, DCM $(5 \times 10 \mathrm{~mL})$, and $\mathrm{MeOH}(5 \times 10 \mathrm{~mL})$. The resins 
were then dried under high vacuum for $\sim 2-4$ hours before the solid-phase coppercatalyzed azide alkyne cycloaddition.

Solid-phase copper-catalyzed azide alkyne cycloaddition was used to incorporate the dynamic covalent functionality onto the ligand by reacting the azido-ligands with alkynes that carry the dynamic covalent bonding pair. The azido-ligand resin and alkyne (4-ethynylbenzaldehyde ( $\mathbf{A L})$ or 4-phenyl-1-butyne (BL); 1.2 eq. per azide) were added to a $20 \mathrm{~mL}$ vial containing $2 \mathrm{~mL}$ DMF. The vial was sealed with a septum and purged with nitrogen gas. In a separate vial a catalytic solution consisting of TBTA (0.4 eq.), sodium ascorbate (0.4 eq.), and copper iodide ( 0.2 eq.) were dissolved in $6 \mathrm{~mL}$ of DMF and $2 \mathrm{~mL}$ of water. The catalytic solution was then added to the resin bound ligand and alkyne and was agitated under $\mathrm{N}_{2}$ at room temperature overnight. The resulting resin was then collected by vacuum filtration and washed with $50 \mathrm{~mL}$ of DMF, $25 \mathrm{~mL}$ of DCM, and $25 \mathrm{~mL}$ of methanol ligands were cleaved with agitation for 2.5 hours using TFA and water (90:10) solution. The resulting cleavage solution was collected, and the TFA was concentrated under flowing $\mathrm{N}_{2}$. The ligand was then precipitated in ether and collected as a white powder.

All ligands were then purified using high performance liquid chromatography (HPLC) and lyophilized before use. HPLC purifications of synthesized ligands were performed on Shimadzu Prominence HPLC system equipped with Zorbax SB-C18 preparatory column $(21.2 \times 250 \mathrm{~mm})$ with $7.0 \mu \mathrm{m}$ packing material. The HPLC gradient conditions were in a binary solvent system of $\mathrm{MeOH}$ ( $1 \%$ formic acid) / water ( $1 \%$ formic acid) $5 \%$ to $90 \% \mathrm{MeOH}$ gradient over 45 minutes. Liquid chromatography mass spectrometry was used to determine the purity and identity of the peak ( $\mathrm{MeOH} /$ water; 5 to $95 \% \mathrm{MeOH}$ gradient over 12 minutes).

Ligand Functionalization Procedure. Nanocrystals in hexane were flocculated using ethanol in excess and centrifuged. The subsequent pellet of nanocrystals was submersed in a solution of AL (0.01 M) in DMF and sonicated for 30 minutes. The nanocrystals were left overnight in solution, and were then flocculated using a hexane:ethanol (1:1) mixture, centrifuged, and dispersed in a neat DMF solution. This was repeated two more times, and the final NC product was dispersed in a solution of neat DMF. ${ }^{19} \mathrm{~F}$ and ${ }^{1} \mathrm{H}$ NMR (Figure S3, and Figure 2) were conducted to confirm the presence of AL on the NCs and, with TGA (Figure S4), quantify the ligand coverage per NC.

Based on NMR, we found an average value of $152 \pm 20$ of ligands per NC. The ligand concentration was found by NMR using a known concentration of fluorchloroform as a reference and comparing to the product peak with the following analysis:

$$
C x=\frac{I x}{I c a l} \times \frac{N c a l}{N x} \times C c a l
$$


$\mathrm{Cx}$ is the concentration of the product, Ix is the integral or area of the peak, and $\mathrm{Nx}$ is the number of expected nucleotides of the product, Ical is the integral or area of the calibrant (spike), Ncal is the number of nucleotides, and Ccal is the concentration (M) of the calibrant.

For TGA the average value of AL per NC was found to be $135 \pm 33$. The reported uncertainties were calculated by propagating the uncertainty in the NMR peak areas and in the measured mass of the NCs.

Nanocrystal Assembly and Disassembly. To assemble the nanocrystals, the nanocrystals were concentrated in a solution of neat DMF $(8 \mathrm{mg} / \mathrm{mL}, 250 \mu \mathrm{L}, 0.4$ $\mathrm{Vol} \%$ ) and separate solutions of oxalyldihydrazide at various linker to nanocrystal number ratios $(\Gamma)$ were made and added to the nanocrystal solutions $(50 \mu \mathrm{L})$ for a final combined solution $(300 \mu \mathrm{L})$ that was monitored for aggregation and gelation.

A control sample was tested with NCs that had no AL present in the ligand shell and were dispersed in DMF (8 mg/mL, $250 \mu \mathrm{L}, 0.4 \mathrm{Vol} \%)$. Linker was added at $\Gamma=200$.

Fourier Transform IR (FTIR) Spectra. FTIR spectral measurements were conducted using a Bruker Vertex $70 \mathrm{FTIR}$ at $4 \mathrm{~cm}^{-1}$ resolution. NC dispersion and $\mathrm{NC}$ gel samples were loaded between two $\mathrm{KBr}$ windows with a spacer $(0.025 \mathrm{~mm})$ using an FTIR liquid cell.

For the fingerprint FTIR spectral measurements, samples were dried on a silicon substrate for 1 week under vaccuum. The samples were background subtracted using bare substrate. The

Dynamic Light Scattering (DLS). Dynamic Light Scattering measurements were performed on a Zetasizer Nano ZS. Samples were diluted $(0.1 \mathrm{mg} / \mathrm{mL})$ with solvent in a quartz cuvette and measured three times integrated for 10 minutes each.

Zeta potential was measured using dilute samples $(1 \mathrm{mg} / \mathrm{mL}, 0.5 \mathrm{mg} / \mathrm{mL}$, and $0.1 \mathrm{mg} / \mathrm{mL}$ ) in a quartz cuvette with a dip cell attachment. Samples were measured three times each and scanned 100 times during each measurement.

Small Angle X-Ray Scattering (SAXS) Analysis. SAXS samples were prepared by injecting a microliter of solution into a capillary tube and flame sealing the sample. SAXS measurements were performed in transmission configuration on the SAXSLAB GANESHA at UT Austin. Measurements were taken 2 days, 1 week, 2 weeks, 4 weeks after preparation. The sample-detector distance was $1 \mathrm{~m}$, and a silver behenate standard was used for calibration. Scattering patterns were converted to $1 \mathrm{D}$ data by circular averaging using the Igor Pro-based Nika software 
for two-dimensional data reduction. SAXS data was background subtracted and analyzed using the Irena tool suite for modeling and analysis in Igor Pro. ${ }^{2,3}$

From the samples the background data was subtracted using neat solutions of the solvent used, and the background subtracted I(q) data was fit using a spheroid form factor from the shoulder of the I(q) data. The form factor fit was then used to divide the Structure factors were extracted from the I(q) by dividing the form factor found from the scattering profile of a dilute nanocrystal dispersion using the Nika software for Igor pro (8). ${ }^{4}$

Scanning Transmission Electron Microscopy (STEM). Samples were prepared by drop-casting NC dispersions onto carbon-coated 400 mesh copper grids, and the imaging was performed in a Hitachi S5500 scanning transmission electron microscope (STEM) operating in the STEM mode at an accelerating voltage of 30 $\mathrm{kV}$. Sizing NCs using the bright-field STEM images with the use of image $\mathrm{j}^{5}$ found $\mathrm{NC}$ sizes to be $12.3 \mathrm{~nm} \pm 1.1 \mathrm{~nm}$.

Thermogravimetric Analysis (TGA). Experiments were performed using Mettler Toledo TGA 2. Samples were loaded onto ceramic crucibles $(12 \mathrm{mg})$ and dried under vacuum for 1 week to remove excess solvent. Samples were heated to $550{ }^{\circ} \mathrm{C}$ at a ramp rate of $20^{\circ} \mathrm{C} / \mathrm{min}$ under air. Analysis of the TGA results was carried out using Igor pro software. A change in the weight as the samples were heated at and above $300{ }^{\circ} \mathrm{C}$ was observed indicating the removal of the organic ligand shell from the NCs (Figure S4).

To calculate the number of ligands per NC the differential weight loss was deconvoluted into contributions from oleate and AL ligands, fitting each with a Gaussian peak shape. The relative contributions of each species and the total weight lost were used to calculate the moles of each ligand type based on the molecular weights. The moles of NCs present was determined based on the final weight of the sample after TGA, the NC size, and the mass density of indium oxide. The ratio of these two quantities is the average number of ligands per NC.

Nuclear Magnetic Resonance (NMR) Spectroscopy. NMR Samples were prepared in a glove box with an inert atmosphere. All NMR solvents were calibrated with their respective solvent peaks. Proton and ${ }^{19} \mathrm{~F}$ NMR spectra were acquired on a MR400 system with a $400 \mathrm{MHz}$ magnet and a OneNMR probe with Protune accessory. NMR spectra were acquired with a relaxation delay of $2 \mathrm{~s}\left(1 \mathrm{~s}\right.$ for $\left.{ }^{19} \mathrm{~F}\right)$, an acquisition time of $4 \mathrm{~s}\left(1 \mathrm{~s}\right.$ for $\left.{ }^{19} \mathrm{~F}\right)$, a total of 64 scans $\left(128\right.$ for $\left.{ }^{19} \mathrm{~F}\right)$ and a pulse angle of $30^{\circ}$. All NMR spectral data was processed with the Mestrenova software package.

To calculate the total amount of ligands per NC using ${ }^{19} \mathrm{~F} N M R$, the samples were spiked with a known concentration of fluorochloroform $(0.03 \mathrm{M})$ and a known amount 
of 4-fluorophenylhydrazine $(0.03 \mathrm{M})$, and the product peak concentration was calculated using the know spike concentration in comparison to the unknown based on peak area. The error in the concentration was determined based on the fitting of each peak and was used to calculate the uncertainty of the ligand count per NC. 
a
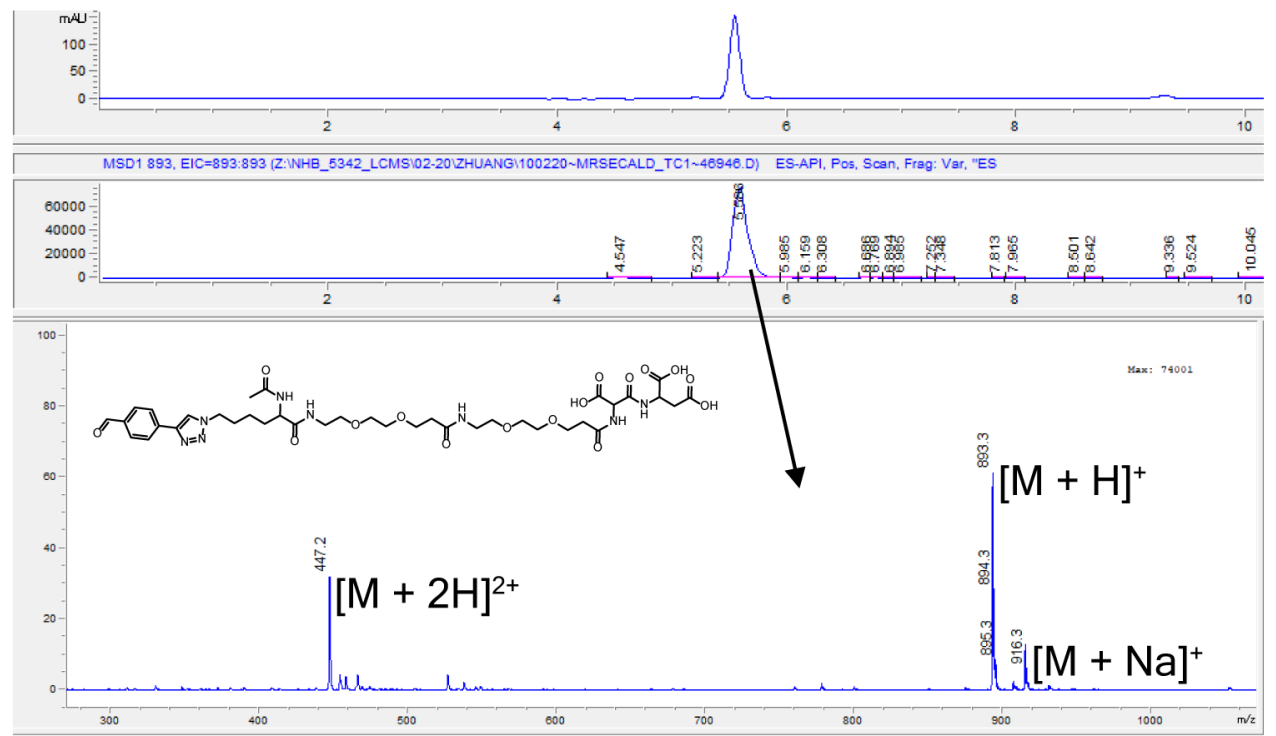

b

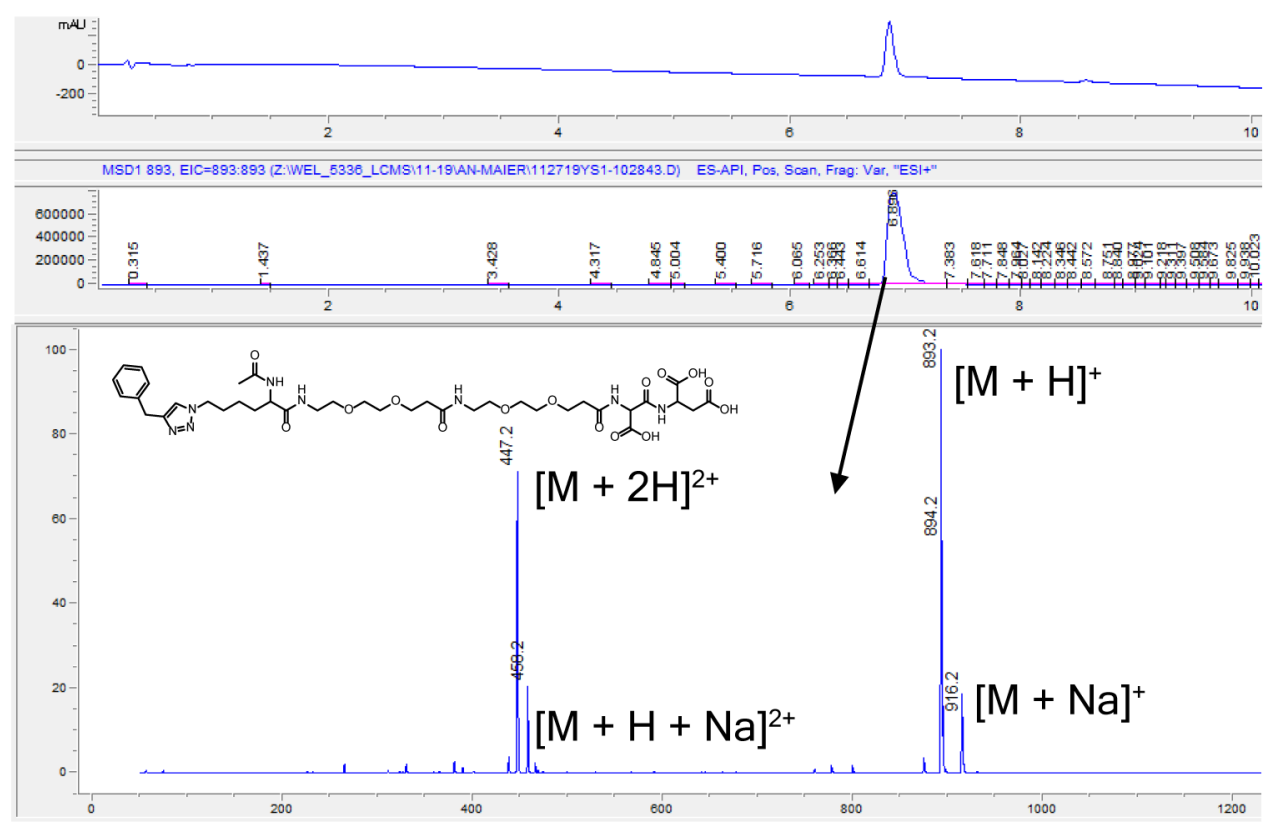

Figure S1. Ligand characterization. Liquid chromatography-mass spectrometry was used to characterize the ligand after peptide synthesis, and to identify the purity of the compound. a) LCMS of the aldehyde derivative ran in a water : methanol gradient from $5 \%$ to $95 \%$ methanol over 12 mins, with $0.5 \%$ formic acid. Extracted ion chromatogram and ESI-MS data of AL b) Similar LCMS of BL under identical conditions. 

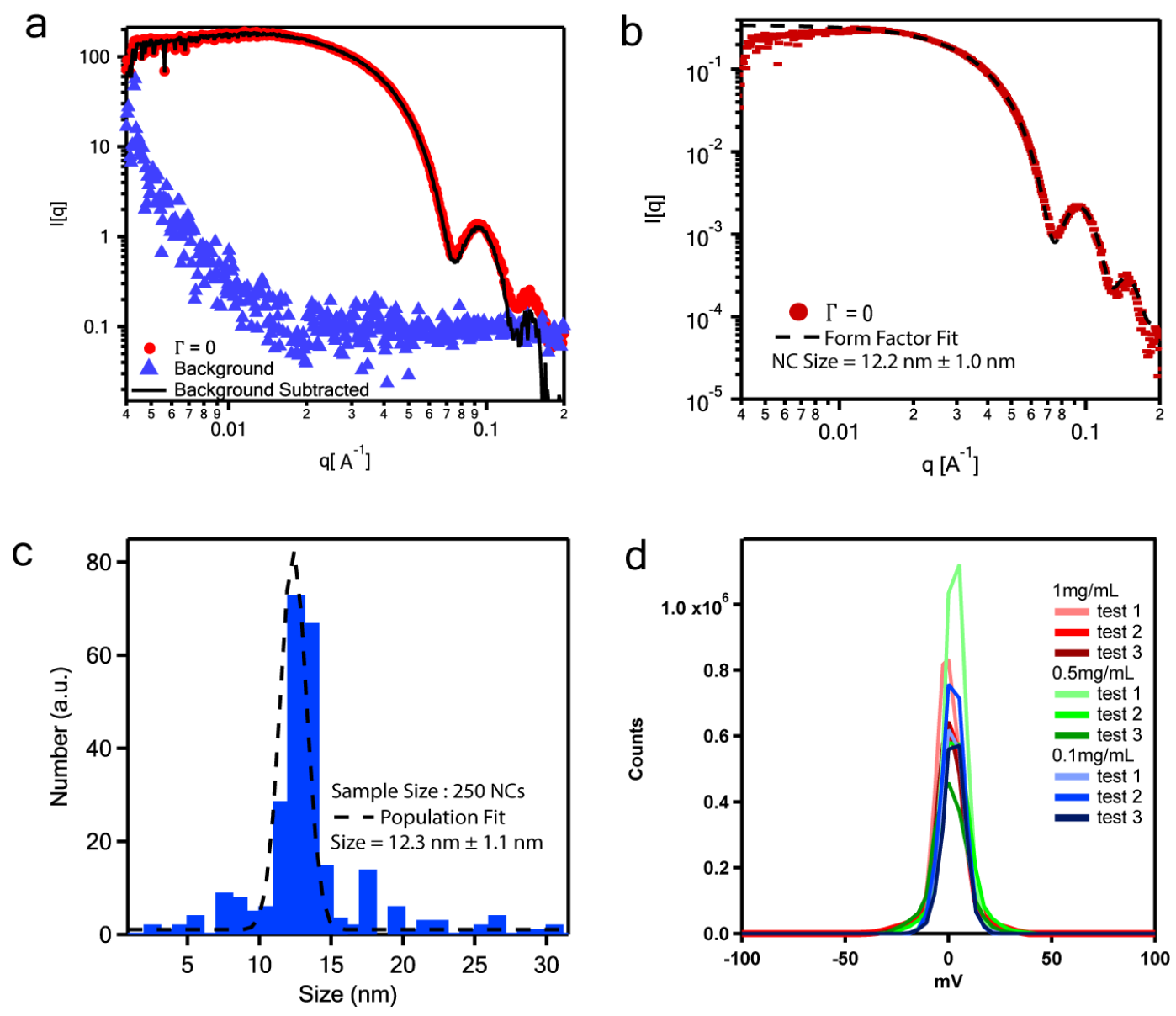

Figure S2. Size and charge analysis of ITO NCs. a) Before fitting, each SAXS pattern was background subtracted using the scattering measured for neat solvent. b) The ITO NC scattering curve was fit with a spheroid form factor, including a Gaussian distribution of sizes, using the high q data points beginning at the shoulder of the form factor $(q=0.03)$. This fitting procedure is further defined in detail in previous literature ${ }^{6,7} \mathrm{c}$ ) Size histogram determined by STEM with a population of $250 \mathrm{NCs}$ and a Gaussian best fit to the distribution. d) Zeta potential of AL-NCs at dilute concentrations was measured as an average $\zeta=$ $+0.03 \mathrm{mV}$. 


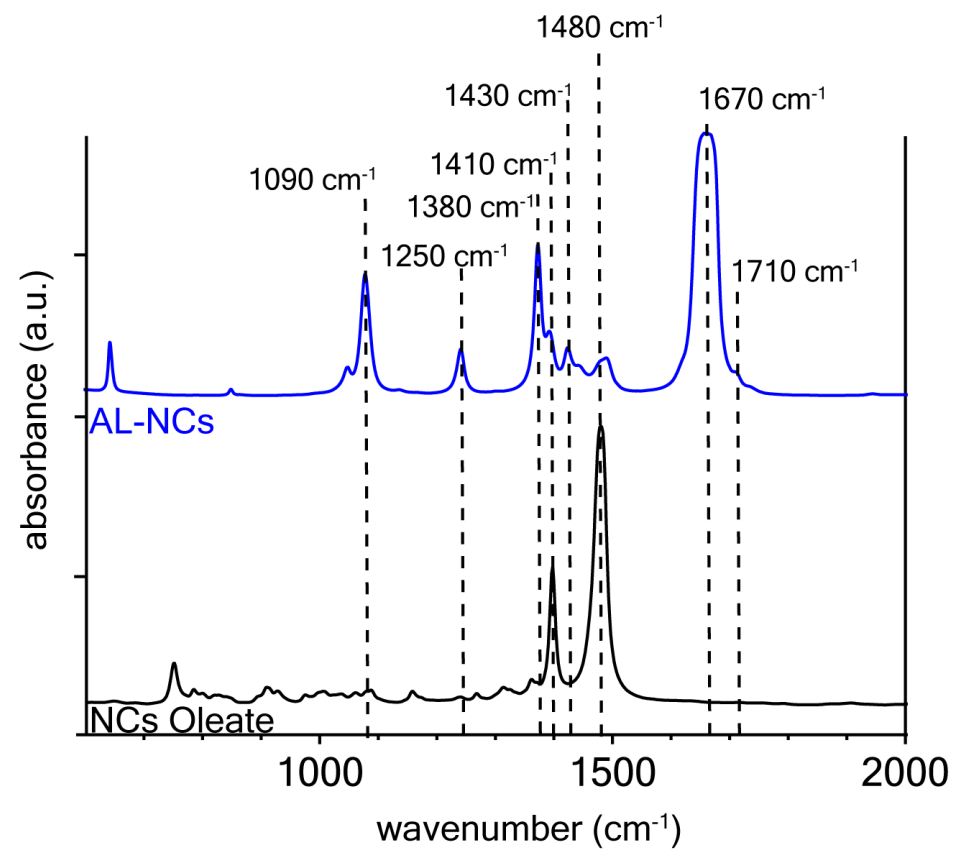

Figure S3. FTIR of NCs before and after functionalization with AL. The oleate carbonyl (1480 $\mathrm{cm}^{-1}$ ) decreases in relative intensity after functionalization. We observe the presence of amide, carboxylic acid, and aldehyde peaks as a broad intense peak $\left(1670 \mathrm{~cm}^{-1}\right.$, shoulder $\left.1710 \mathrm{~cm}^{-1}\right)$, any residual DMF may also contribute. Aldehyde bending peaks are observed near carboxylic acid bending peaks $\left(1380 \mathrm{~cm}^{-1}, 1430 \mathrm{~cm}^{-1}\right.$ in the AL NCs and $1410 \mathrm{~cm}^{-1}$ for oleic acid). The peak associated with the ethylene glycol backbone $\left(1090 \mathrm{~cm}^{-1}\right)$ and another amine stretch $\left(1250 \mathrm{~cm}^{-1}\right)$ are seen for the AL-NCs. 
a<smiles>CC(C)c1ccc(C=O)cc1</smiles><smiles>NNc1ccc(F)cc1</smiles><smiles></smiles><smiles>CCc1ccc(/C=N/Nc2ccc(F)cc2)cc1</smiles>

b

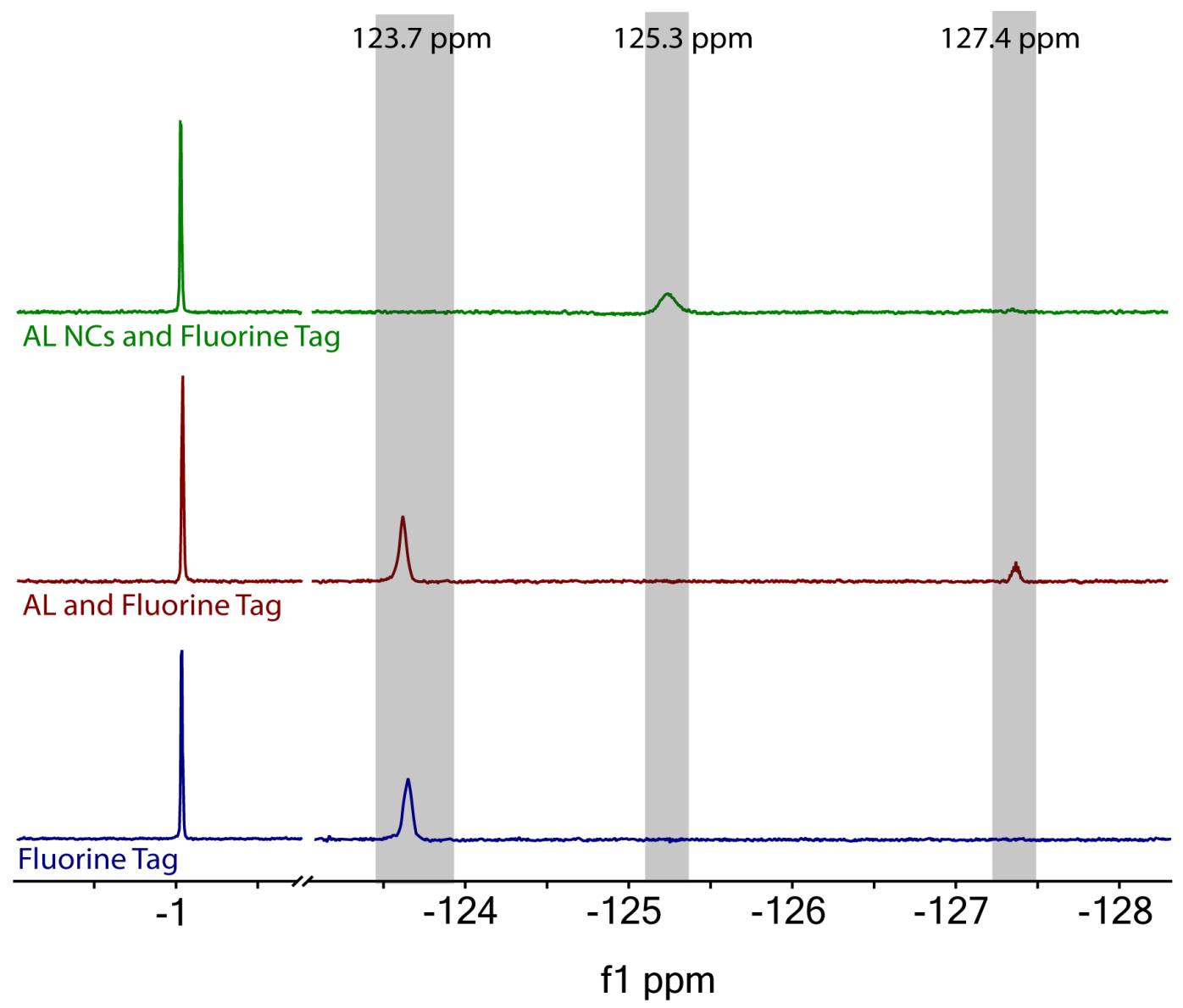

Figure S4. ${ }^{19} \mathrm{~F}-\mathrm{NMR}$ was used to quantify the number surface ligands bound to the NCs. a) The fluorine-tag molecule, 4-fluorophenylhydrazine, was introduced in excess to a solution of AL-NCs $(8 \mathrm{mg} / \mathrm{mL})$. A similar AL-only solution was prepared to identify the peak with no NCs present. The peak at $123.7 \mathrm{ppm}$ is associated with free fluorine-tag molecules. A slight shift is detected in the presence of the ligand, and a secondary peak at $127.4 \mathrm{ppm}$ associated with bound F-tag emerges. When NCs are present, the bound peak shifted to $125.3 \mathrm{ppm}$ and broadened. 

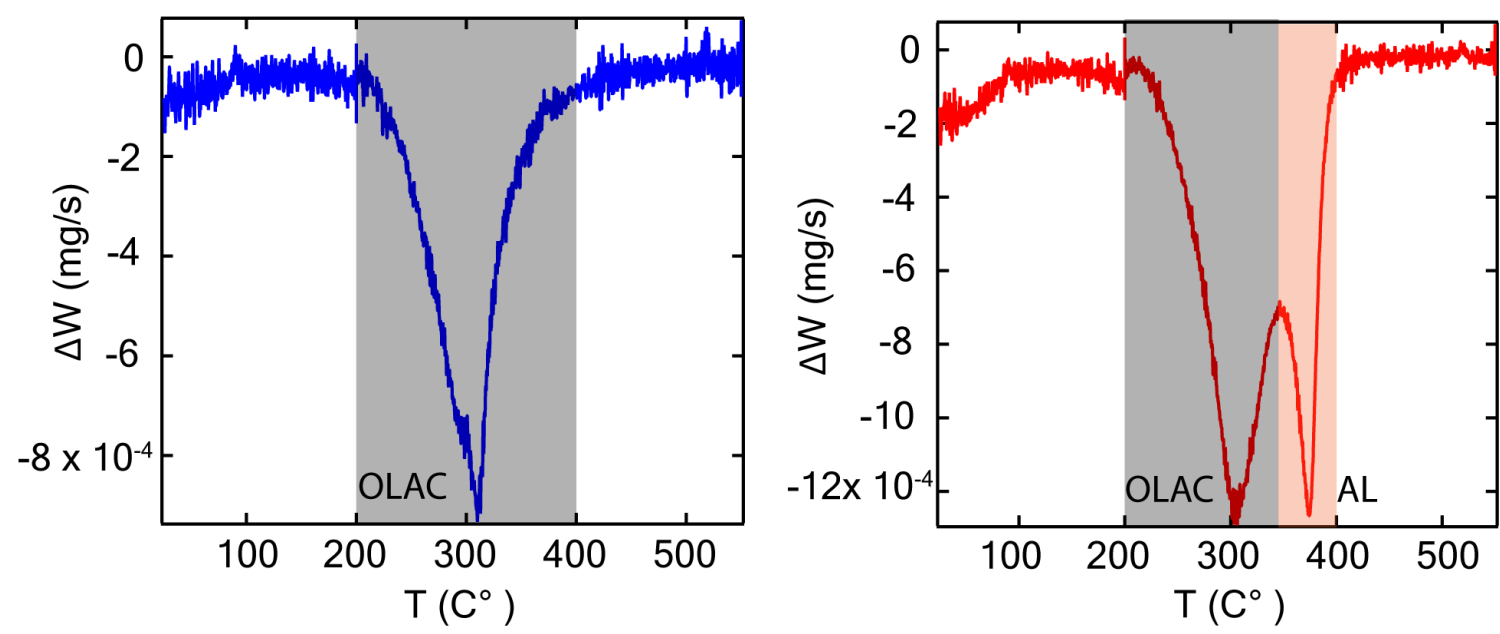

Figure S5. Differential mass determined by TGA of NCs capped with oleic acid and AL ligands used to identify the number of ligands present on the NCs, for comparison with ${ }^{19} \mathrm{~F}-\mathrm{NMR}$. We observed in the oleic acid cap NCs a peak around $300{ }^{\circ} \mathrm{C}$ indicating the removal of oleate ligands from our NCs. The two distinct peaks found for AL-NCs were deconvoluted to determine the number of AL ligands per NC. 


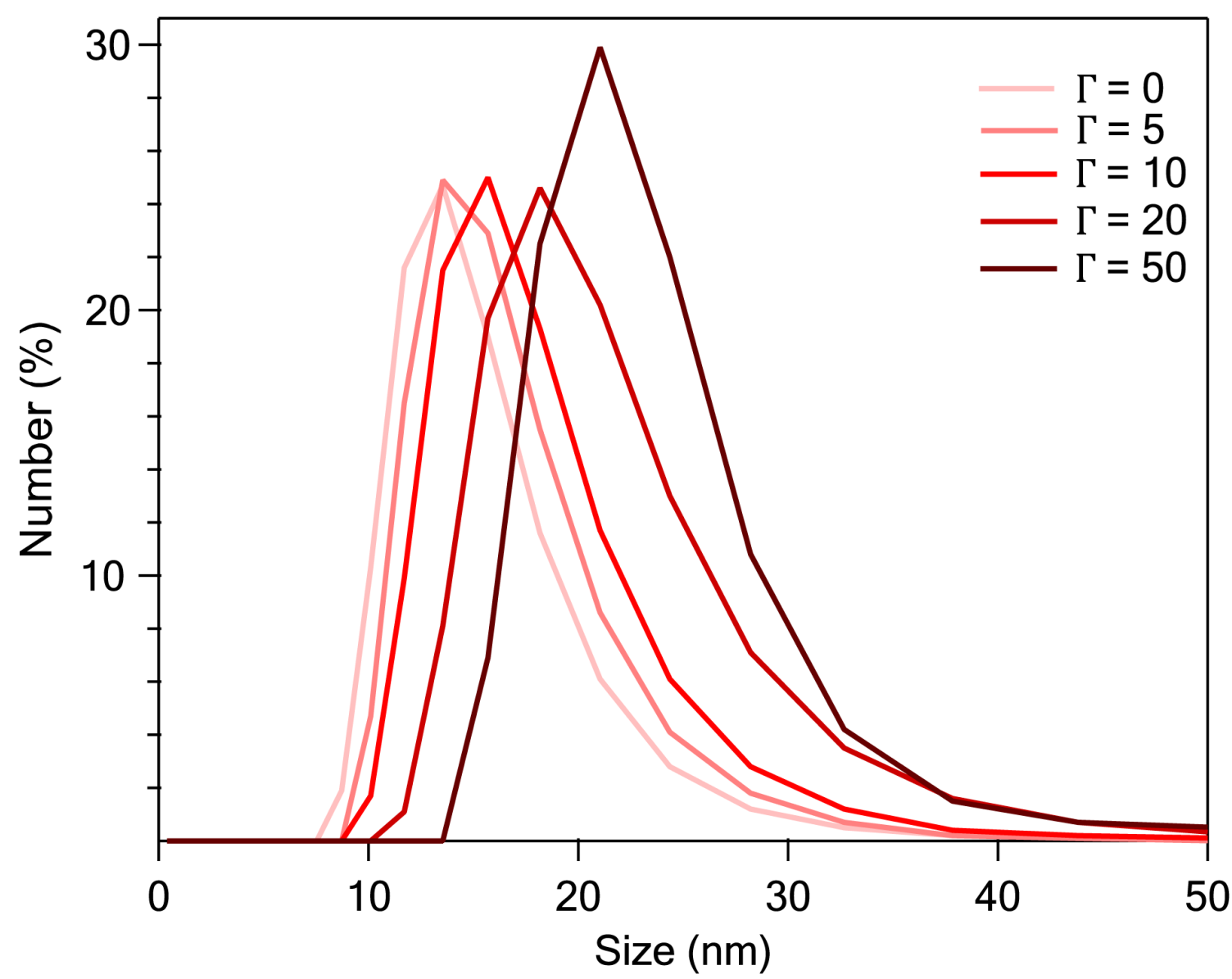

Figure S6. Dynamic light scattering of samples from $\Gamma=1$ to $\Gamma=50$ approximately 1 day after introducing OD linker to the AL-NC dispersion. As the linker concentration increased, the average hydrodynamic diameter determined by DLS increased, indicating NC aggregation. Very small changes were observed until $\Gamma=50$, where appreciable NC clustering was observed. 


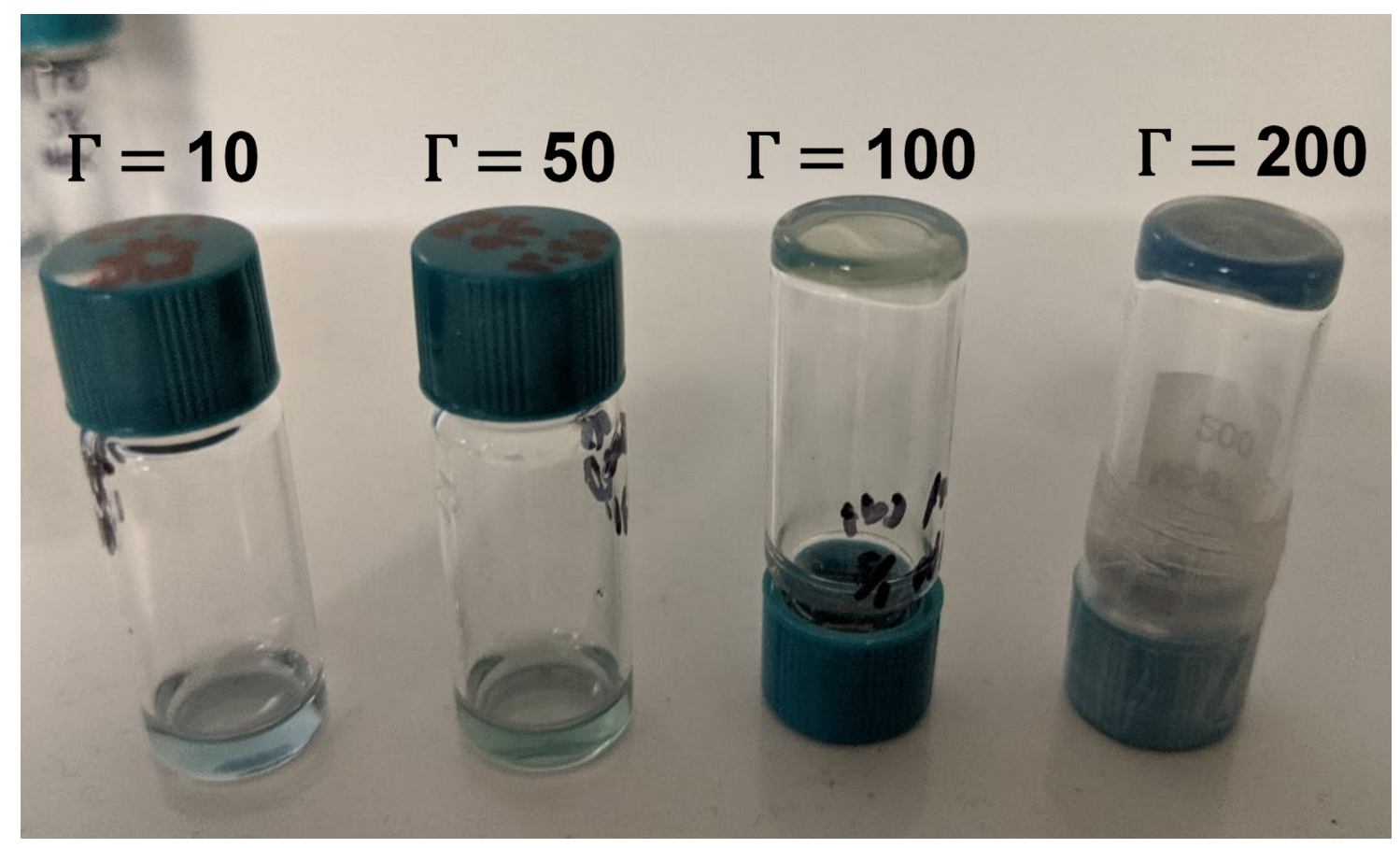

Figure S7. Photographs of NC samples 1 month after adding OD linker. Dispersions with low linker concentrations, $\Gamma=10$ and $\Gamma=50$, remain flowing while those at large linker concentrations, $\Gamma=100$ and $\Gamma=200$, form two phases and the gels are self-supporting (the second, dilute flowing phase is not visible in the photographs). 

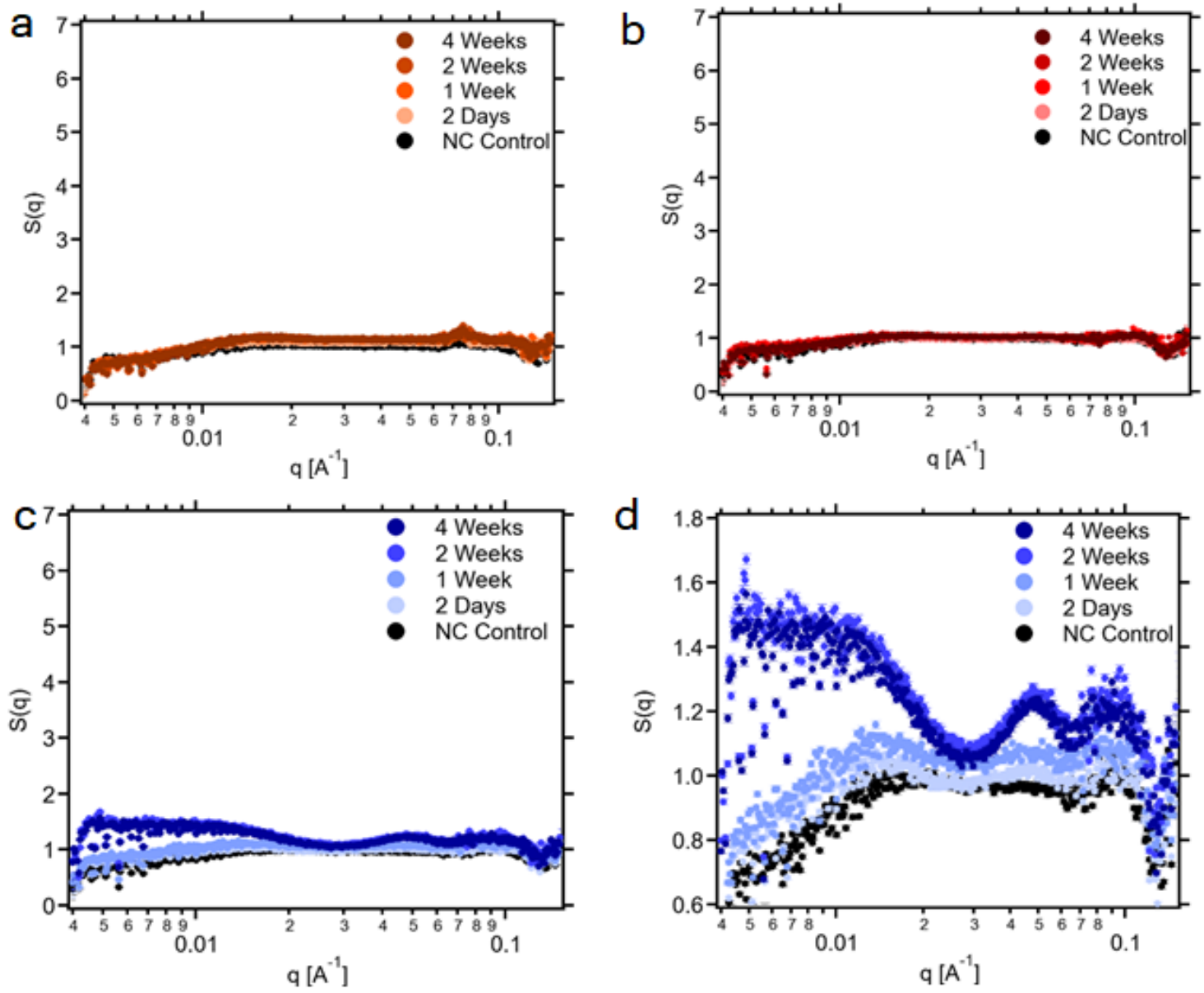

Figure S8. SAXS structure factor (S(q)) of samples for various linker-to-NC ratios of a) $\Gamma=3 b$ ) $\Gamma$ $=10 \mathrm{c}) \Gamma=50$ and d) close up of $\Gamma=50$. In each case, the NC control sample has no linker added. 
a

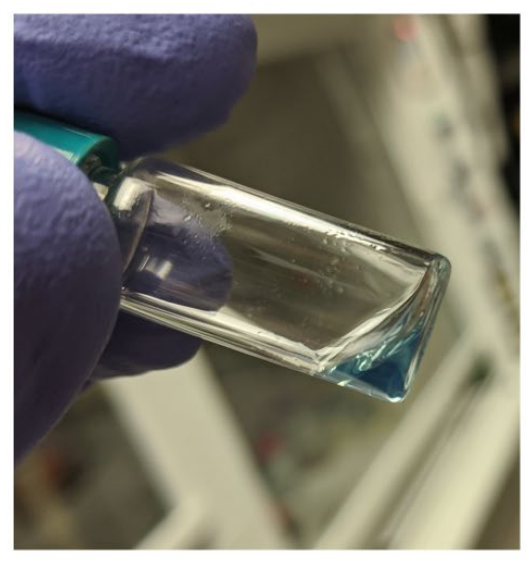

b

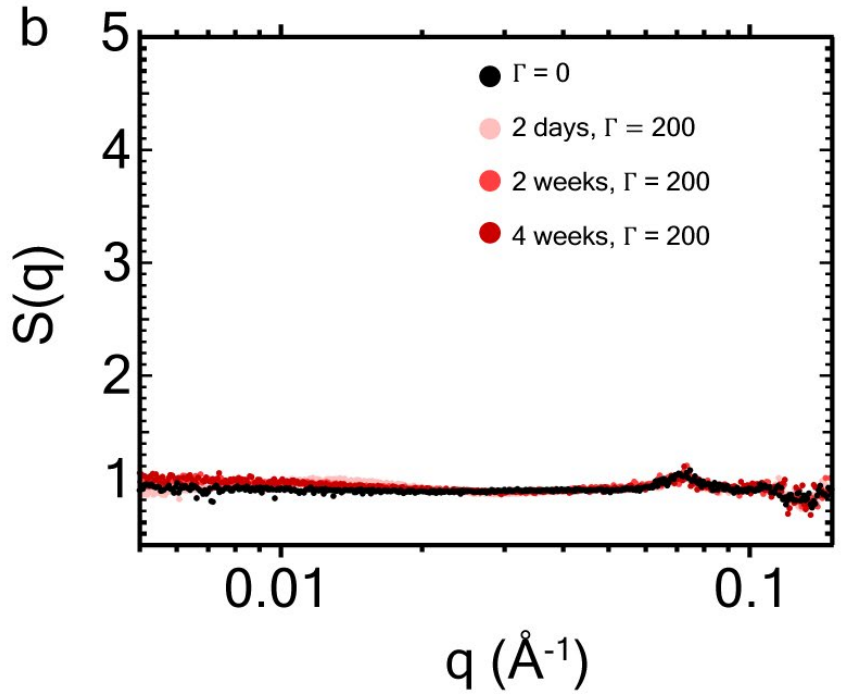

Figure S9. Photo and SAXS of a control experiment (described in assembly methods) using NCs functionalized with $B L$ at $\Gamma=200$. a) Shows the dispersion after 2 days, which appears still well dispersed. b) S(q) also indicates little change over 2 days when compared to the NC dispersion and in comparison to reported AL-NC samples (Figure 3c,d). 

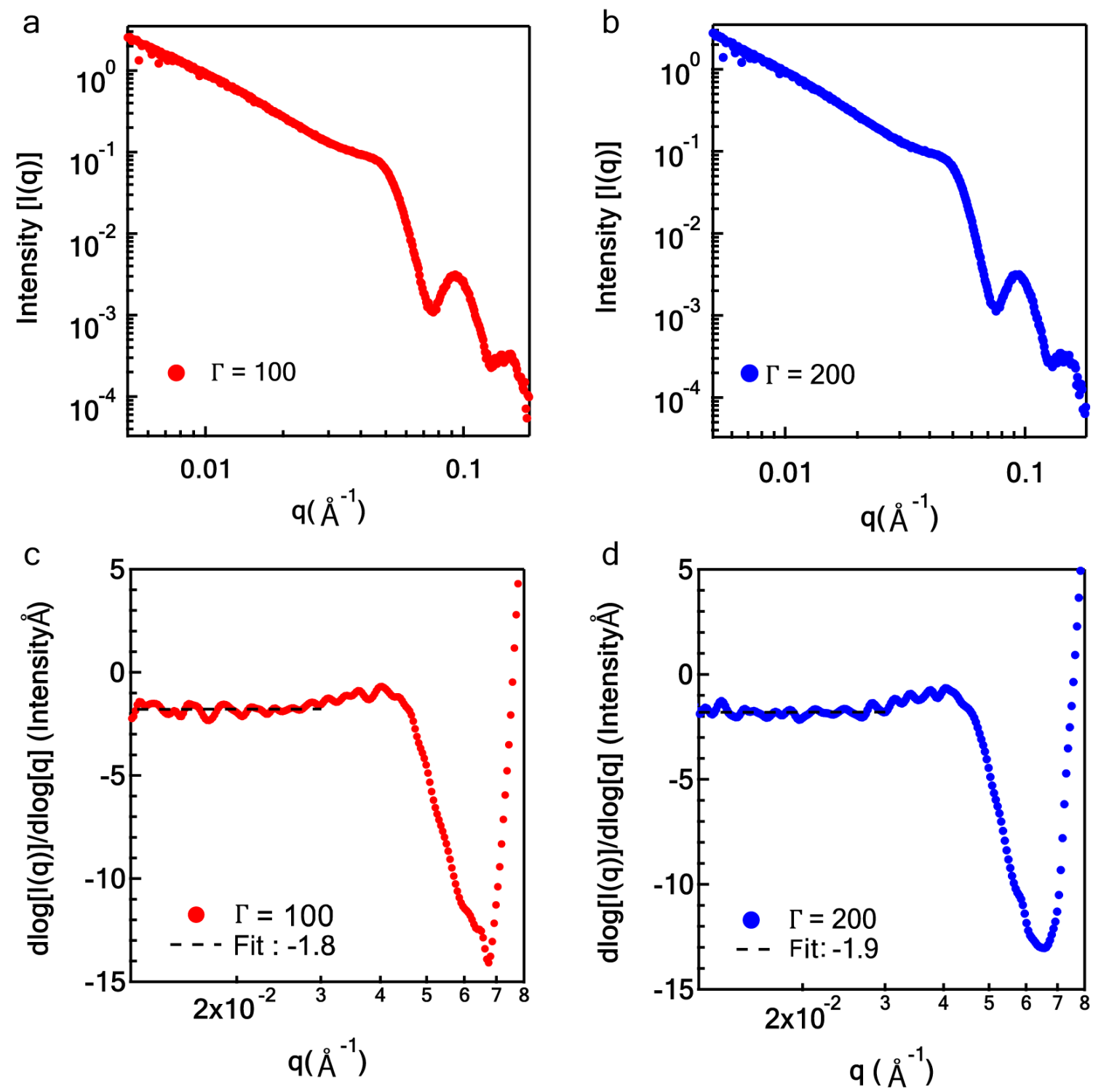

Figure S10. The background subtracted $\mathrm{I}(\mathrm{q})$ was used to find the $\mathrm{q}$ range for the Porod region and to determine the fractal dimension $\left(D_{f}\right)$ in the $\Gamma=100(a)$ and $\Gamma=200(b)$ systems. A single Porod region is apparent where the differential intensity is approximately constant $(c, d)$. The slope of $\log (\mathrm{I}(\mathrm{q}))$ vs $\log (\mathrm{q})$ was found by fitting to a power law equation $\left[\mathrm{I}(\mathrm{q})=\mathrm{B} \mathrm{q}^{-\mathrm{Df}]}\right.$. $^{8}$ 


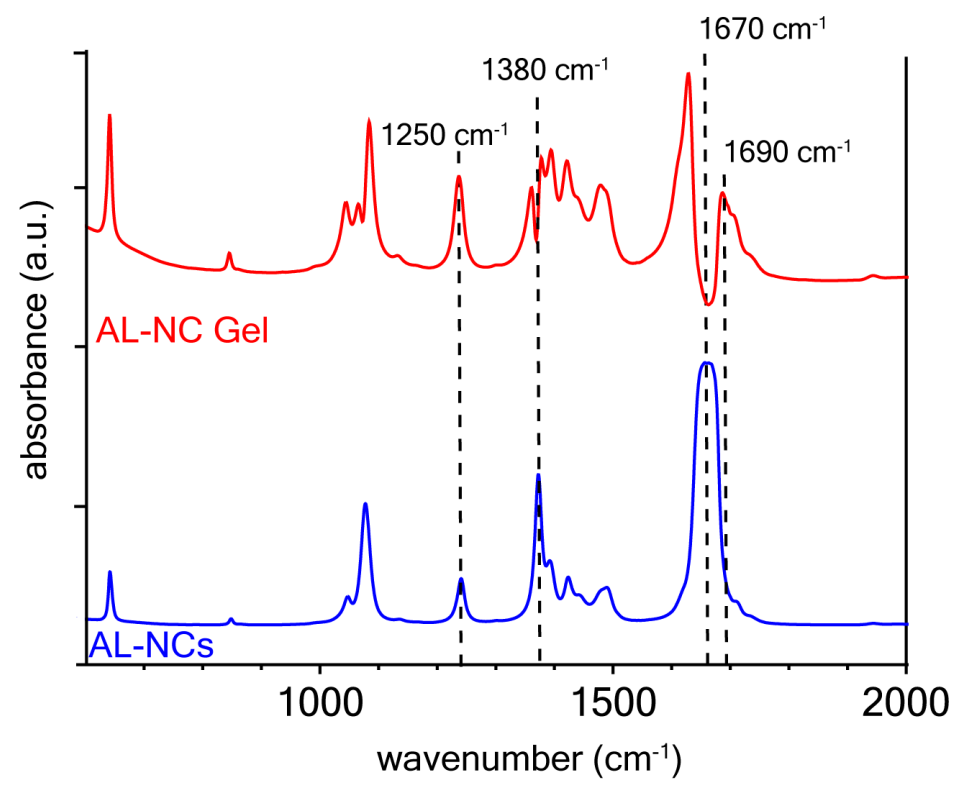

Figure S11. FTIR of an AL-NC gel $(\Gamma=200)$, and a dispersed phase of AL-NCs. The imine peak associated with the hydrazone product is present in the gel data at $1690 \mathrm{~cm}^{-1}$ and an increase of the amine stretch at $1250 \mathrm{~cm}^{-1}$ due to the presence of the OD is apparent. A decrease in the aldehyde bending at $1380 \mathrm{~cm}^{-1}$ indicates the loss of these groups by conversion to hydrazone. 
a

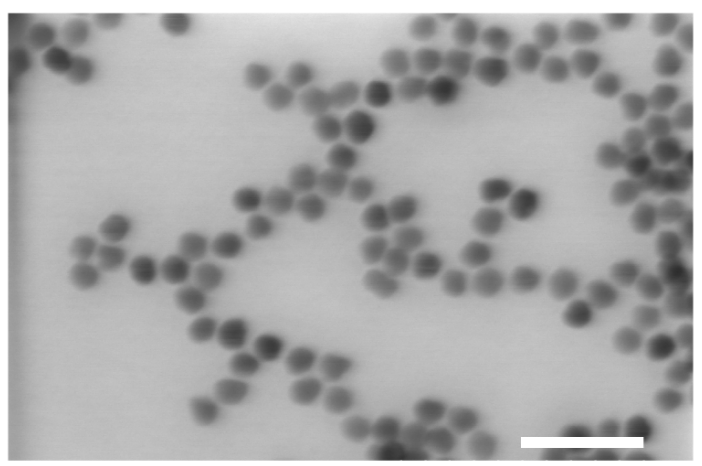

C

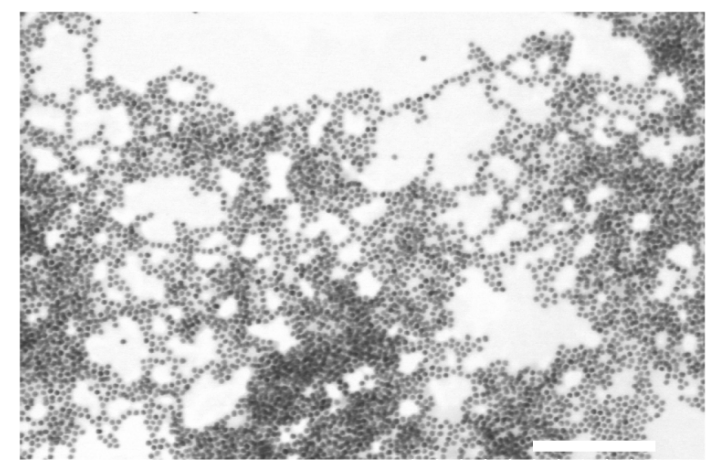

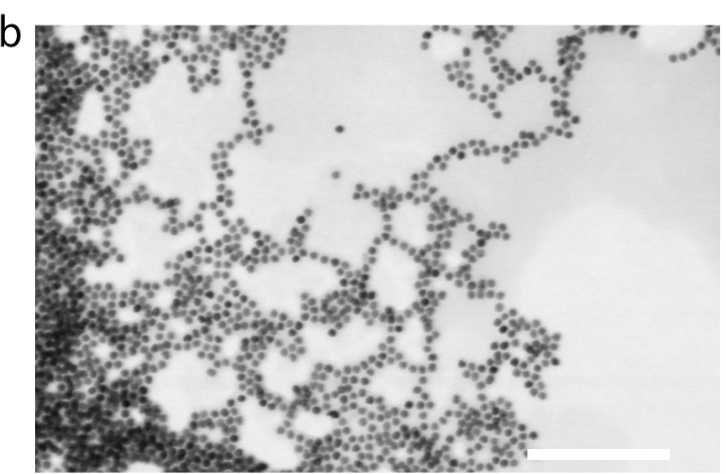

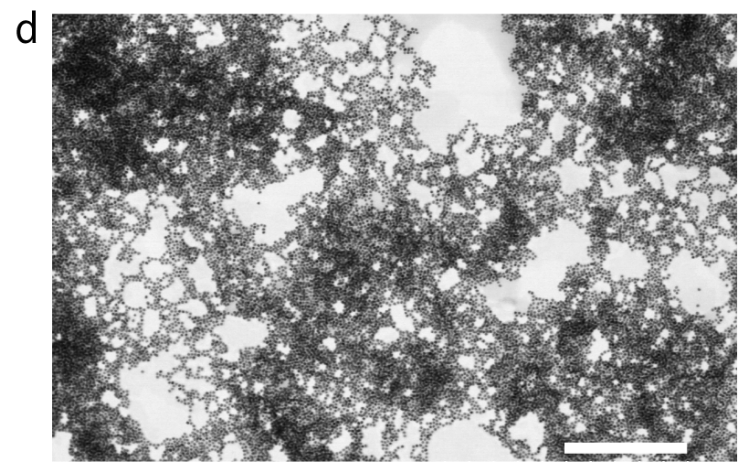

Figure S12. STEM of assembled particles showing stringy gel networks. Locally, the NCs are densely clustered in some regions, but they form an open, porous structure on larger length scales. Scale bars are a) $50 \mathrm{~nm} \mathrm{b)} 200 \mathrm{~nm}$ c) $250 \mathrm{~nm}$ d) $500 \mathrm{~nm}$. 


\section{THEORETICAL MODEL AND METHODS}

Model. We developed a simplified physical model for the NC-linker mixture based on both our previous theoretical efforts ${ }^{9,10}$ and a recently published model for the gelation of DNA nanostars. ${ }^{11}$ As a significant assumption, we neglected any attractions between the NCs or their ligands, which is equivalent to assuming that the NCs and ligands have only short-ranged steric repulsions and are well dispersed in the absence of the linker. We modeled the ligands as linear bead-spring chains and set the diameter of a bead to $\sigma=0.7 \mathrm{~nm}$, which is roughly the Kuhn length for the polyethylene oxide-like groups in the ligands. ${ }^{12}$ We estimated the end-to-end length of the ligand as roughly $5 \mathrm{~nm}$, so each ligand was 7 beads in length. Each NC was represented as a single larger sphere having diameter $\sigma_{\mathrm{NC}}=12.2 \mathrm{~nm}$. Based on our ${ }^{19} \mathrm{~F}-\mathrm{NMR}$ measurement, we attached 154 ligands to each NC's surface by randomly choosing permanent attachment sites from the 642 vertices of an icosphere ${ }^{13}$ having radius $\left(\sigma_{\mathrm{NC}}+\sigma\right) / 2$. A linker molecule was approximated as a sphere of roughly equal size to a segment of the ligand (diameter $\sigma$ ).

Nonbonded pairwise interactions between particles representing the NCs, ligands, and linkers were modeled using the core-shifted Lennard-Jones potential,

$$
u_{\mathrm{nb}}\left(r_{i j}\right)=4 \varepsilon\left[\left(\frac{\sigma}{r_{i j}-\delta_{i j}}\right)^{12}-\left(\frac{\sigma}{r_{i j}-\delta_{i j}}\right)^{6}\right],
$$

where $r_{i j}$ is the center-to-center distance between two particles $i$ and $j$ and $\delta_{i j}=$ $\left(\sigma_{i}+\sigma_{j}\right) / 2-\sigma$ shifts the divergence of the potential to account for the different diameters of the particles, $\sigma_{i}$ and $\sigma_{j}$. We set the energy scale $\varepsilon$ for the repulsion equal to the thermal energy, $\varepsilon=k_{\mathrm{B}} T$, with $k_{\mathrm{B}}$ being Boltzmann's constant and $T$ being the temperature. The potential was truncated and shifted to zero at its minimum, $r_{i j}^{*}=2^{1 / 6} \sigma+\delta_{i j}$, to render these interactions purely repulsive. Taken together, these parameters create a model for nearly hard spheres. ${ }^{14}$

Bonded pairs of ligand particles interacted as harmonic springs,

$$
u_{\mathrm{b}}\left(r_{i j}\right)=\frac{\kappa_{\mathrm{b}}}{2}\left(r_{i j}-b_{0}\right)^{2},
$$

where $\kappa_{\mathrm{b}}=600 k_{\mathrm{B}} T / \sigma^{2}$ sets the bond stiffness and $b_{0}=0.96 \sigma$ is the equilibrium bond length. This value of $b_{0}$ gives roughly the same average bond length as the Kremer-Grest polymer model ${ }^{15}$ and so, in conjunction with $u_{\mathrm{nb}}$, should prevent unphysical chain crossing.

To mimic dynamic covalent bonding between the ligand and linker molecules, we introduced complementary attractive patches to these molecules. ${ }^{11}$ One "A" patch 
was added to the free end of each ligand, while two "B" patches were added to each linker. The patches were placed on the surface of the ligand or linker particle using a harmonic spring (eq S2) having $b_{0}=2^{1 / 6} \sigma / 2 \approx 0.561 \sigma$. Additionally, the angle $\theta$ between the ligand patch and the ligand's penultimate bead and/or between the two patches on a linker molecule was restrained to $180^{\circ}$ ( $\pi$ radians) using a harmonic bending potential,

$$
u_{\theta}(\theta)=\frac{\kappa_{\theta}}{2}(\theta-\pi)^{2},
$$

where $\kappa_{\theta}=600 k_{\mathrm{B}} T$ is the bending stiffness. We added complementary (A-B) attraction and same-type (A-A and $B-B)$ repulsion between patches to mimic the formation of an exclusive bond between one A patch and one B patch. Specifically, all patches interacted through the standard Lennard-Jones potential (similar to eq S1 with $\delta_{i j}=0$ ),

$$
u_{i j}\left(r_{i j}\right)=4 \varepsilon_{i j}\left[\left(\frac{\sigma_{i j}}{r_{i j}}\right)^{12}-\left(\frac{\sigma_{i j}}{r_{i j}}\right)^{6}\right]
$$

where $\varepsilon_{i j}$ and $\sigma_{i j}$ set the energy and length scales for the interactions. Figure S13 schematically illustrates the complete model.

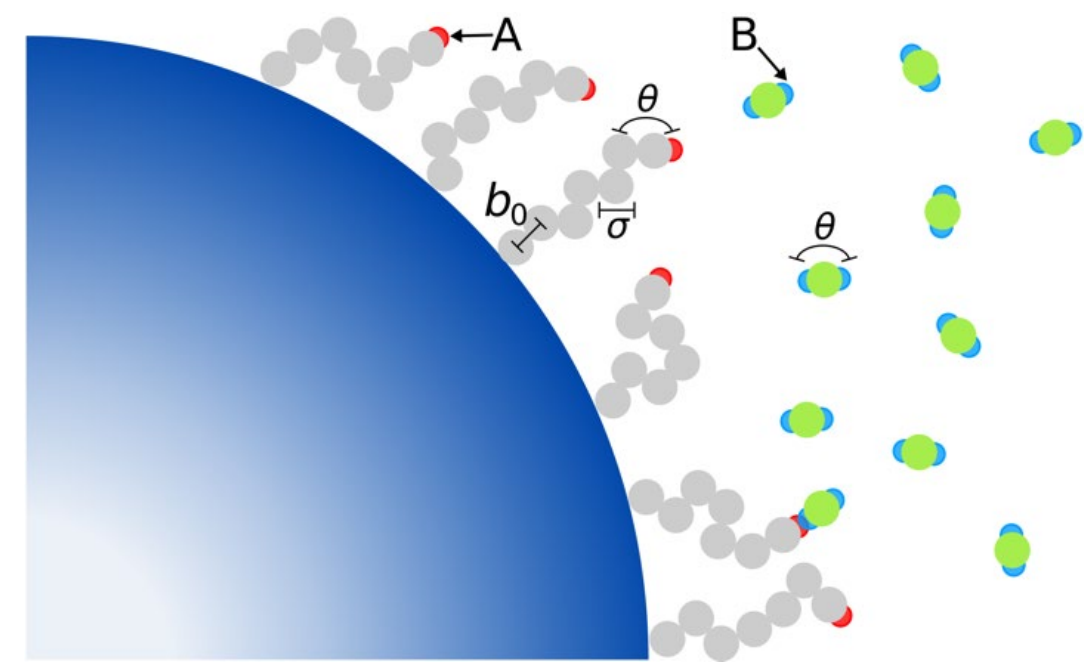

Figure S13. Schematic of a NC (dark blue) with its ligands (grey), the linkers (green), and the A (red) \& B (light blue) patches. The size of a ligand bead $\sigma$, the length of a ligand bead-ligand bead bond $b_{0}$, and the patch angles $\theta$ are also indicated. 
We set $\sigma_{\mathrm{AB}}=0.2 \sigma$ and truncated these complementary attractions at $5 \sigma_{\mathrm{AB}}=1.0 \sigma$. We then set $\sigma_{\mathrm{AA}}=\sigma_{\mathrm{BB}}=2 / 3 \sigma$ and truncated these same-type repulsions at $2^{1 / 6} \sigma_{\mathrm{AA}} \approx 0.748 \sigma$. The larger values of $\sigma_{\mathrm{AA}}$ and $\sigma_{\mathrm{BB}}$ relative to $\sigma_{\mathrm{AB}}$ prevent, e.g., multiple $B$ patches from coordinating with the same $A$ patch. We chose $\varepsilon_{\mathrm{AA}}=\varepsilon_{\mathrm{BB}}=$ $k_{\mathrm{B}} T$ as for the other repulsions in the model, but the attraction strength $\varepsilon_{\mathrm{AB}}$ should be connected to the strength of a typical covalent bond. For the studied chemistry, this bond dissociation energy is expected to be orders of magnitude larger than the thermal energy. However, such large values of $\varepsilon_{A B}$ would unacceptably decrease the timestep required to faithfully integrate the equations of motion in a molecular simulation of this model. Our prior work indicated that the phase behavior of similar nanoparticle-linker mixtures became insensitive to the value of $\varepsilon_{\mathrm{AB}}$ provided that it was sufficiently larger than $k_{\mathrm{B}} T ; 10$ we accordingly chose $\varepsilon_{\mathrm{AB}}=20 k_{\mathrm{B}} T$ as the attraction strength.

Theory. To rationalize the experimentally observed gelation behavior, we computed theoretical spinodal boundaries for the NC-linker mixture with respect to the NC volume fraction $\eta_{\mathrm{NC}}=\pi \sigma_{\mathrm{NC}}^{3} n_{\mathrm{NC}} / 6$ and linker-to-NC ratio $\Gamma=n_{\text {link }} / n_{\mathrm{NC}}$. Here, $n_{\mathrm{NC}}$ and $n_{\text {link }}$ are the number densities of the NCs and linkers, respectively. The spinodal demarcates the region in which the single-phase (fluid) mixture is thermodynamically unstable to composition fluctuations and will spontaneously separate into two phases, one of which will enrich in NCs and tend to arrest as a gel. In the canonical ensemble (constant temperature and volume), this region can be found using the determinant of the stability matrix $\mathbf{H}$ that comprises mixed partial derivatives of the Helmholtz free-energy density $f$ with respect to composition, $H_{i j}=\partial^{2} f / \partial n_{i} \partial n_{j}$.

For the free energy $f$, we use the Chapman, Jackson, and Gubbins formulation ${ }^{16}$ of Wertheim's first-order thermodynamic perturbation theory (TPT). ${ }^{17,18}$ The free energy is decomposed into two terms: the free energy of a reference fluid $f_{0}$ (NCs, ligands, and linkers) that does not form bonds between components and the free energy due to formation of bonds between the ligands and the linkers $f_{\mathrm{b}}$. TPT makes several approximations to yield a simple expression for $f_{\mathrm{b}}$, perhaps the most significant of which are that each bond formation event is independent and that there are no cycles in the graph representing the bonded NC-linker network. The consequences of these assumptions can be tested directly by molecular simulation (see Simulations).

For the reference fluid, we used a polyatomic hard-chain equation of state, ${ }^{19}$ itself derived from TPT,

$$
\beta f_{0}=\beta f^{\mathrm{id}}+\beta f_{\mathrm{hs}}^{\mathrm{ex}}-\sum_{i} n_{i} \sum_{j-k \text { bonds }} \ln g_{\mathrm{hs}}\left(\sigma_{j k}^{+}\right)
$$


where $\beta=1 / k_{\mathrm{B}} T, f^{\mathrm{id}}$ is the free energy of an ideal mixture of chains, and $f_{\mathrm{hs}}^{\mathrm{ex}}$ is the excess free energy of the hard-sphere mixture that would be obtained by dissolving all bonds from the chains. The last term is the excess free energy due to chain formation; the outer sum runs over the chain types $i$, while the inner sum runs over bonds between particles of types $j$ and $k$ within the chain. We neglected volume excluded by the patches, so the nanoparticle-ligand complex is considered a branched chain with two types of bonds (154 NC-ligand bonds, $154 \times 6=924$ ligand bead-ligand bead bonds), while the single-bead linker has no bonds. $g_{\mathrm{hs}}\left(\sigma_{j k}^{+}\right)$ is the value of the radial distribution function at contact between the particles, i.e., $\sigma_{j k}^{+}=\left(\sigma_{j}+\sigma_{k}\right) / 2$. We used Boublík's equation of state for the hard-sphere mixture ${ }^{20}$ to calculate $f_{\mathrm{hs}}^{\mathrm{ex}}$ and $g_{\mathrm{hs}}\left(\sigma_{j k}^{+}\right)$using the nominal diameters of each particle in the model.

For the bonding between ligands and linkers, TPT gives the free energy of bonding for complementary "patchy" components, ${ }^{16}$

$$
\beta f_{\mathrm{b}}=\sum_{i} n_{i} \gamma_{i}\left[\ln X_{i}-\frac{1-X_{i}}{2}\right],
$$

where $\gamma_{i}$ is the number of patches (all of the same type) on component $i$ and $X_{i}$ is the fraction of those patches that are not bonded. We set the number of patches per $\mathrm{NC}$ equal to the number of ligands $\left(\gamma_{\mathrm{NC}}=154\right)$, while the number of patches per linker molecule was $\gamma_{\text {link }}=2$. The values of $X_{i}$ are determined by

$$
X_{i}=\left[1+\sum_{j} n_{j} \gamma_{j} X_{j} \Delta_{i j}\right]^{-1},
$$

where $\Delta_{i j}$ is an integral over the Mayer $f$-function for the patches on $i$ and $j$ and is given by ref 16 . For our model, $\Delta_{\mathrm{NC}-\mathrm{NC}}=\Delta_{\text {link-link }}=0$ because their patches are purely repulsive. We approximate the cross-term $\Delta_{\mathrm{NC} \text {-link }}$ assuming the pair correlations between particles are given by their dissociated hard-sphere contact value,

$$
\Delta_{\mathrm{NC}-\mathrm{link}} \approx 4 \pi \sigma^{2} g_{\mathrm{hs}}\left(\sigma^{+}\right) \int \mathrm{d} r\left\langle e^{-\beta u_{\mathrm{AB}}\left[r_{\mathrm{AB}}\left(r, \omega_{A}, \omega_{B}\right)\right]}-1\right\rangle_{\omega_{\mathrm{A}}, \omega_{\mathrm{B}}} .
$$

The brackets denote an unweighted average over angular orientations of the patches $\omega_{\mathrm{A}}$ and $\omega_{\mathrm{B}}$ at a fixed separation $r$ of the particles to which they are attached; this integral must be taken numerically. We first used Monte Carlo sampling to take the ensemble average $\left(10^{8}\right.$ configurations per separation) at particle separations 
$1.0 \sigma \leq r \leq 2.0 \sigma$ (21 points, uniform spacing) for which the integrand is nonzero, and we then used the trapezoidal rule to integrate with respect to $r$.

To find the spinodal boundary, we computed the stability matrix $\mathbf{H}$ numerically using a finite-difference scheme for the partial derivatives of $f$. We first performed a grid search in $0.002 \leq \eta_{\mathrm{NC}} \leq 0.02$ (37 points, uniform spacing) and $1 \leq \Gamma \leq 1000$ (60 points, $\log _{10}$ spacing) to find ranges of $\Gamma$ where $|\mathbf{H}|$ changed sign for a fixed $\eta_{\mathrm{NC}}$, and we then used Brent's method to solve $|\mathbf{H}|=0$ within this bounded range. Figure $4 a$ shows this locus of solutions, with the fluid phase being reentrant with respect to $\Gamma$; state points between the two curves are thermodynamically unstable. In this work, we focus on the low- $\Gamma$ branch that is most relevant to our experiments.

Simulations. We noted that the theoretically predicted spinodal boundary deviated significantly from our experimental observations; gelation occurred at significantly higher linker-to-NC ratios than predicted (Figure 4a). We hypothesized that this deviation was due to the formation of cycles ("loops") in the NC-linker network, in violation of one of TPT's key assumptions. To test this hypothesis, we performed molecular simulations of the NC-linker mixture using LAMMPS (22 Aug 2018). ${ }^{21} \mathrm{We}$ randomly dispersed $100 \mathrm{NCs}$ in a cubic triply periodic simulation box with edge length $L \approx 410 \sigma \quad\left(\eta_{\mathrm{NC}}=0.004\right)$. We considered linker-to-NC ratios $\Gamma=$ $10,50,100$, and 200 and randomly inserted the linkers without overlaps. (The total number of particles in the simulations ranged from 126300 to 183300.) We used a ligand particle as the nominal unit mass $m$; the linker and patch particle were also given unit mass, while the NC particle mass was scaled by its volume, $m_{\mathrm{NC}}=$ $\left(\sigma_{\mathrm{NC}} / \sigma\right)^{3} m \approx 5294 \mathrm{~m}$. The interactions were the same as those described in Model, and the equations of motion were integrated using a velocity Verlet scheme with timestep $0.0025 \tau$ coupled to a Nosé-Hoover thermostat with time constant $0.1 \tau$, where $\tau=\sqrt{\beta m \sigma^{2}}$ is the unit of time. The NCs and the first bead of each of their attached ligands were treated as rigid bodies that were coupled to a separate, identical thermostat. This integration scheme implies artificial dynamics neglecting, e.g., solvent drag on the NCs; we found this was necessary to observe aggregation during the accessible simulation times.

We initially equilibrated the NC-linker mixtures for $5 \times 10^{4} \tau$ with purely repulsive interactions between all patches. We then switched on the complementary attractions between $A$ and $B$ patches and simulated for $1.5 \times 10^{6} \tau$, quickly observing linkers attaching to ligands. We monitored the number and size of NC clusters that formed; two NCs were considered to be in the same cluster if they were connected by a linker. We also measured the number of linkers forming different types of links between ligands or NCs. These measures are shown in Figure $\mathbf{5}$ and discussed in detail in the main text. Although the mixture was far from equilibrium when the 
attraction was initially switched on, we found that both the largest cluster size and the number of links approached a steady value by the end of the simulations.

We additionally performed calculations and simulations where the number of functional ligands on the surface of the NC was reduced (Figures S14, S15, and S16). We achieved this in TPT by reducing $\gamma_{\mathrm{NC}}$ and in the simulations by introducing an additional patch type " $C$ " that had purely repulsive interactions with both the "A" and "B" patches. We then randomly selected ligands to have either " $A$ " or " $C$ " patches based on the desired level of functionalization.

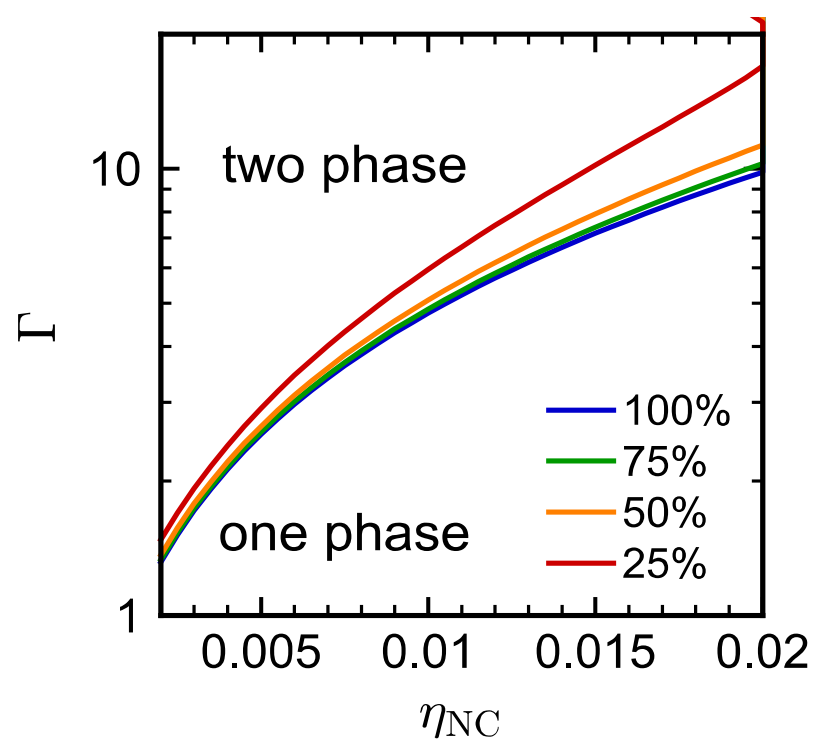

Figure S14. Low- $\Gamma$ branch of the spinodal boundary for NC-linker mixtures with varying percentages of functionalized ligands. 

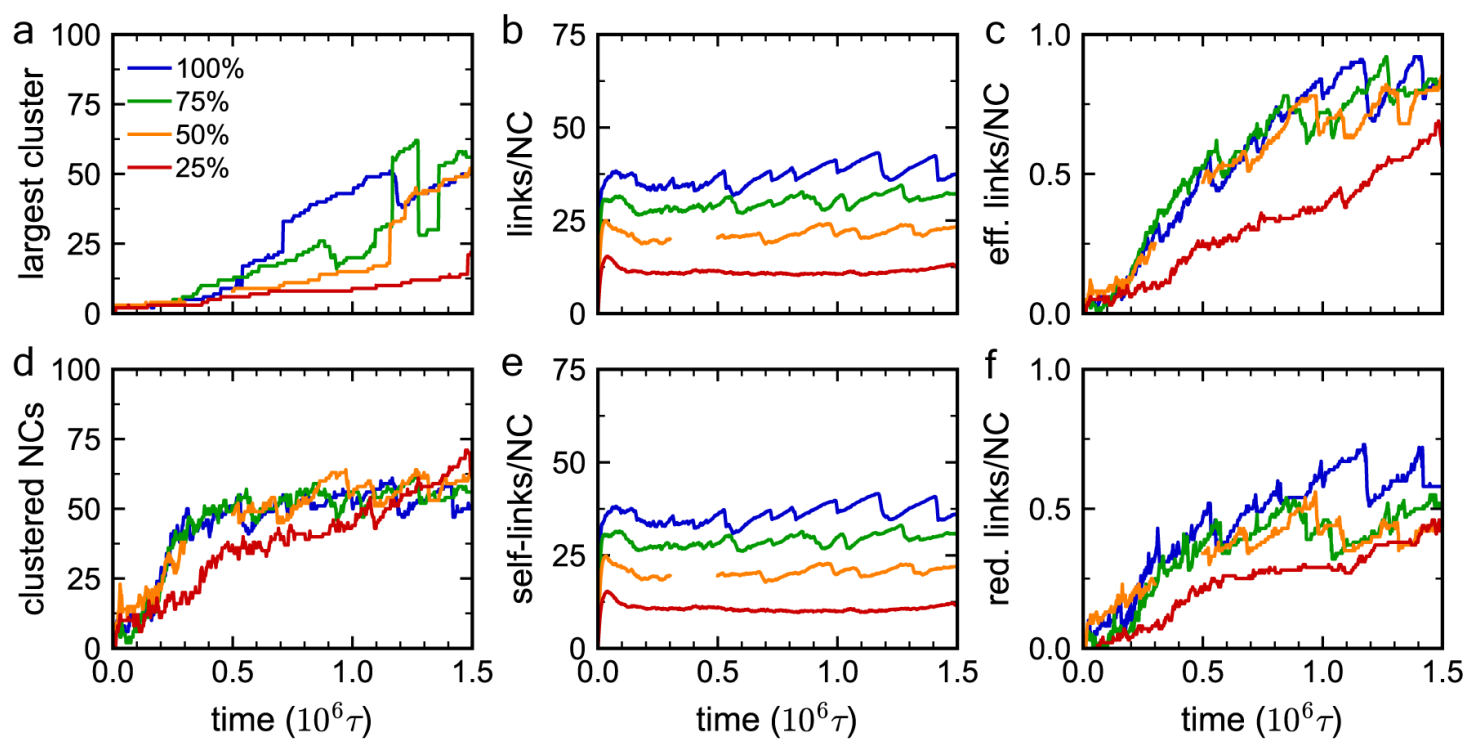

Figure S15. Analysis of simulated NC-linker structures with limited NC functionalization. (a) Largest NC cluster size and (d) total number of NCs in clusters as a function of simulation time for varied fractions of functionalized ligands when $\Gamma=50$. Number of linkers (per NC) forming (b) a link between any two ligands, (c) an effective (unique) link between two NCs, (e) a self-link between two ligands on the same NC, and (f) a redundant link between two NCs. The total number of NCs is 100. 

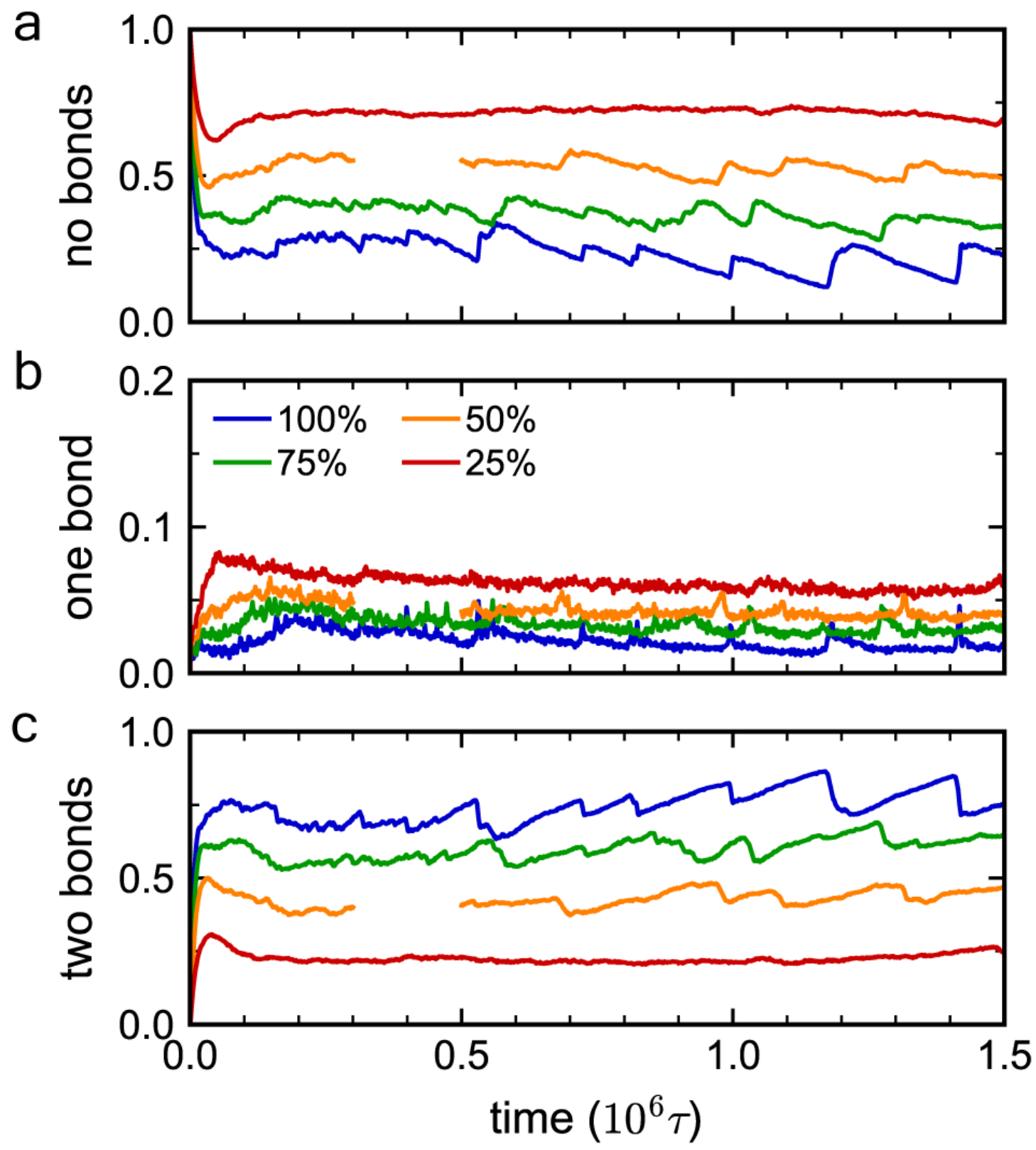

Figure S16. Fraction of linkers with different numbers of bonds for NCs with limited functionalization. The linkers with (a) no bonds are "free," the linkers with (b) one bond have capped a ligand without forming a link, and the linkers with (c) two bonds form the links in

Figure S15b. The linker-to-NC ratio is $\Gamma=50$, and the total number of NCs is 100 , as in Figure S15. 


\section{REFERENCES}

1 Jansons, A. W.; Hutchison, J. E. Continuous Growth of Metal Oxide Nanocrystals: Enhanced Control of Nanocrystal Size and Radial Dopant Distribution. ACS Nano 2016, 10, 6942-6951.

${ }^{2}$ Hexemer, A.; Bras, W.; Glossinger, J.; Schaible, E.; Gann, E.; Kirian, R.; MacDowell, A.; Church, M.; Rude, B.; Padmore, H. A SAXS/WAXS/GISAXS Beamline with Multilayer Monochromator. J. Phys. Conf. Ser. 2010, 247, 12007.

3 Ilavsky, J.; Jemian, P. R. Irena: Tool Suite for Modeling and Analysis of SmallAngle Scattering. J. Appl. Crystallogr. 2009, 42, 347-353.

4 Ilavsky, J. Nika: Software for Two-Dimensional Data Reduction. J. Appl. Crystallogr. 2012, 45, 324-328.

${ }^{5}$ Schneider, C. A.; Rasband, W. S.; Eliceiri, K. W. NIH Image to Image J: 25 Years of Image Analysis. Nat. Methods 2012, 9, 671-675.

${ }^{6}$ Singh, A.; Lindquist, B. A.; Ong, G. K.; Jadrich, R. B.; Singh, A.; Ha, H.; Ellison, C. J.; Truskett, T. M.; Milliron, D. J. Linking Semiconductor Nanocrystals into Gel Networks through All-Inorganic Bridges. Angew. Chemie Int. Ed. 2015, 54, 1484014844.

${ }^{7}$ Saez Cabezas, C. A.; Ong, G. K.; Jadrich, R. B.; Lindquist, B. A.; Agrawal, A.; Truskett, T. M.; Milliron, D. J. Gelation of Plasmonic Metal Oxide Nanocrystals by Polymer-Induced Depletion Attractions. Proc. Natl. Acad. Sci. 2018, 115, 89258930.

${ }^{8}$ Beaucage, G. Small-Angle Scattering from Polymeric Mass Fractals of Arbitrary Mass-Fractal Dimension. J. Appl. Crystallogr. 1996, 29, 134-146.

${ }^{9}$ Lindquist, B. A.; Jadrich, R. B.; Milliron, D. J.; Truskett, T. M. On the Formation of Equilibrium Gels via a Macroscopic Bond Limitation. J. Chem. Phys. 2016, 145, 74906.

${ }^{10}$ Howard, M. P.; Jadrich, R. B.; Lindquist, B. A.; Khabaz, F.; Bonnecaze, R. T.; Milliron, D. J.; Truskett, T. M. Structure and Phase Behavior of Polymer-Linked Colloidal Gels. J. Chem. Phys. 2019, 151, 124901.

${ }^{11}$ Xing, Z.; Ness, C.; Frenkel, D.; Eiser, E. Structural and Linear Elastic Properties of DNA Hydrogels by Coarse-Grained Simulation. Macromolecules 2019, 52, 504512. 
12 Lee, H.; Venable, R. M.; Mackerell Jr, A. D.; Pastor, R. W. Molecular Dynamics Studies of Polyethylene Oxide and Polyethylene Glycol: Hydrodynamic Radius and Shape Anisotropy. Biophys. J. 2008, 95, 1590-1599.

${ }^{13}$ Swan, J. W.; Wang, G. Rapid Calculation of Hydrodynamic and Transport Properties in Concentrated Solutions of Colloidal Particles and Macromolecules. Phys. Fluids 2016, 28, 11902.

14 Weeks, J. D.; Chandler, D.; Andersen, H. C. Role of Repulsive Forces in Determining the Equilibrium Structure of Simple Liquids. J. Chem. Phys. 1971, 54, 5237-5247.

${ }^{15}$ Everaers, R.; Karimi-Varzaneh, H. A.; Fleck, F.; Hojdis, N.; Svaneborg, C. Kremer-Grest Models for Commodity Polymer Melts: Linking Theory, Experiment, and Simulation at the Kuhn Scale. Macromolecules 2020, 53, 1901-1916.

${ }^{16}$ Chapman, W. G.; Jackson, G.; Gubbins, K. E. Phase Equilibria of Associating Fluids. Mol. Phys. 1988, 65, 1057-1079.

17 Wertheim, M. S. Fluids with Highly Directional Attractive Forces. III. Multiple Attraction Sites. J. Stat. Phys. 1986, 42, 459-476.

${ }^{18}$ Wertheim, M. S. Fluids with Highly Directional Attractive Forces. IV. Equilibrium Polymerization. J. Stat. Phys. 1986, 42, 477-492.

${ }^{19}$ Amos, M. D.; Jackson, G. Bonded Hard-sphere (BHS) Theory for the Equation of State of Fused Hard-sphere Polyatomic Molecules and Their Mixtures. J. Chem. Phys. 1992, 96, 4604-4618.

${ }^{20}$ Boublík, T. Hard-Sphere Equation of State. J. Chem. Phys. 1970, 53, 471-472.

${ }^{21}$ Plimpton, S. Fast Parallel Algorithms for Short-Range Molecular Dynamics. J. Comput. Phys. 1995, 117, 1-19. 